Disponível em: http://periodicos.ufpb.br/ojs2/index.php/recfin

\title{
ASSOCIAÇÃO DA EVIDENCIAÇÃO DO GERENCIAMENTO DE RISCOS COM GOVER- NANÇA CORPORATIVA E DESEMPENHO EM EMPRESAS COM ADRs ${ }^{1}$
}

\section{ASSOCIATION BETWEEN RISKS MANAGEMENT DISCLOSURE AND CORPORATE GOV- ERNANCE AND PERFORMANCE IN COMPANIES WITH ADRS}

\author{
Iago Franca Lopes \\ Doutorando em Ciências Contábeis (UFPR) \\ Universidade Federal do Paraná \\ iagofrancalopes@gmail.com \\ Ilse Maria Beuren \\ Doutora em Ciências Contábeis (USP) \\ Universidade Federal de Santa Catarina \\ ilse.beuren@gmail.com \\ Ernesto Fernando Rodrigues Vicente \\ Doutor em Administração (USP) \\ Universidade Federal de Santa Catarina \\ ernesto.vicente@ufsc.br
}

\section{RESUMO}

Objetivo: Este estudo analisa a associação da evidenciação do gerenciamento de riscos com governança corporativa e desempenho em empresas brasileiras com American Depositary Receipts (ADRs).

Fundamento: Os investidores consideram informações de risco em suas decisões, assim a evidenciação das ações sobre o gerenciamento de riscos deve estar alinhada à atividade operacional e pode aumentar o desempenho organizacional e conduzir a relação entre organização e sociedade por meio da governança corporativa.

Método: A amostra consistiu de 37 empresas não financeiras. O Índice de Evidenciação da Gestão de Riscos foi apurado mediante a leitura dos Relatórios da Administração e o uso de uma escala dicotômica, seguida de aplicação da técnica da entropia da informação. Os dados para governança corporativa (direito de controle e diferença entre direito de controle e direito sobre o fluxo de caixa do acionista controlador) e desempenho empresarial (Margem Bruta, Retorno sobre o Patrimônio Líquido e Ativo Total) foram extraídos da Economática ${ }^{\circledR}$.

Resultados: As empresas Copel, Sabesp, Petrobras, Tractebel Energia, BRF Foods, Cemig e CESP apresentaram os maiores índices médios de evidenciação no período. $O$ índice de evidenciação da

\footnotetext{
${ }^{1}$ Artigo recebido em: 30/04/2020. Revisado por pares em: 30/09/2020. Reformulado em: 14/10/2020. Recomendado para publicação: 30/09/2020 por Marco Aurélio dos Santos (Editor Adjunto). Publicado em: 11/02/2021. Organização responsável pelo periódico: UFPB
} 
gestão de riscos mostrou-se associado com o ativo total das empresas ao nível de $5 \%$. Já a configuração narrativa sobre divulgação de gerenciamento de riscos nos Relatórios da Administração não se mostrou associada à estrutura de governança corporativa das empresas com ADRs e nem ao desempenho destas no período analisado.

Contribuição: A pesquisa fornece um mapeamento da evidenciação de gerenciamento de riscos das empresas com ADRs. Ademais, sugere que as variáveis de governança corporativa e desempenho empresarial devem ocupar a agenda de pesquisadores, visto que possuem características que contribuem para a consolidação do mercado de capitais brasileiro, além de minimizar a assimetria informacional entre as empresas.

Palavras-chave: Evidenciação. Gestão de riscos. Governança corporativa. Desempenho empresarial.

\begin{abstract}
Objective: This study aims to analyze the association between risks management disclosure and corporate governance and business performance in Brazilian companies with American Depositary Receipts (ADRs).

Fundamentals: Investors consider risk information in their decisions, so the disclosure of actions on risk management must be aligned with operational activity and can increase organizational performance and drive the relationship between the organization and society through corporate governance.

Method: The sample consisted of 37 non-financial companies. The Index of the Risks Management Disclosure was determined by reading the Management Reports and afrom a dichotomous scale, followed by the application of the information entropy technique. The data for corporate governance (control right and difference between control right and the right to control the cash flow of the controlling shareholder) and business performance (Gross Margin, Return on Equity and Total Assets) were extracted from Economática®.

Results: The companies Copel, Sabesp, Petrobras, Tractebel Energia, BRF Foods, Cemig and CESP had the highest average disclosure rates in the period. The risk management disclosure index was associated with the total assets of companies at the level of $5 \%$. The narrative configuration about disclosure of risk management in the Management Reports did not show to be associated with the corporate governance structure of companies with ADRs or with their performance in the analyzed period.

Contributions: The survey provides an mapping of the risk management disclosure of companies with ADRs. Furthermore, it is suggests that the variables of corporate governance and business performance should occupy the agenda of researchers, since they have characteristics that contribute to the consolidation of the Brazilian capital market, in addition to minimizing the information asymmetry between companies.
\end{abstract}

Keywords: Disclosure. Risks management. Corporate governance. Business performance.

\title{
1. INTRODUÇÃO
}

A evidenciação sobre o gerenciamento de riscos surge como um equalizador em resposta aos escândalos de corrupção e fracassos de empresas, que eram consideradas economicamente rentáveis e promissoras (Saito \& Schiozer, 2007; Arena, Arnaboldi \& Azzone, 2010). Estes fatores motivaram o mundo dos negócios a buscar mecanismos para mitiga-los (Arena et al., 2010). Gordon, Loeb e Tseng (2009) apontam que ao se adotar uma sistemática de gerenciamento e controle dos riscos, busca-se mitigar os riscos inerentes à atividade operacional e aumentar a performance empresarial.

Discutir essa temática frente ao mercado de capitais e à comunidade científica mostra-se relevante. Na perspectiva do mercado de capitais, Campbell, Chen, Dhaliwal, Lu e Steele (2014) destacam que os investidores podem incorporar informações de risco em suas decisões de preços e assim melhorar a liquidez do mercado, reduzindo a assimetria de informações. Além disso, em 
cenários de crise e de adversidade é nos relatórios sobre riscos que os investidores buscam informações para subsidiar suas decisões de investimento, o que, por consequência, pode alterar o seu valor no mercado (Miihkinen, 2013). Jin, Chen, Wang, Yu e Long (2020) apontam que, diante de cenários econômicos diversos, a divulgação de informações possibilita ao participante deste mercado uma melhor análise do cenário no qual a organização está inserida e o processo de decisão torna-se mais justo. A divulgação de informações proporciona vantagens aos participantes desse processo e transparência, eleva o grau de concorrência e promove a alocação justa dos recursos para o desenvolvimento da organização (Holmberg \& Wolak, 2016; Jin et al., 2020).

Na perspectiva teórica, Elshandidy e Neri (2015) argumentam que poucos trabalhos examinaram a comunicação de riscos e que, por vezes, está associada à governança corporativa e ao desempenho da empresa no mercado de capitais. Em âmbito internacional (Okafor \& Ibadin, 2011; Miihkinen, 2013; Elshandidy \& Neri, 2015; Jin, Chen, Wang, Yu \& Long, 2020) e nacional (Santos \& Coelho, 2018) algumas pesquisas tangenciaram esta questão. Por exemplo, Okafor e Ibadin (2011) analisaram a relação da divulgação do gerenciamento de riscos com governança corporativa e desempenho corporativo de empresas cotadas na Bolsa de Valores da Nigéria. Os resultados da pesquisa mostraram que governança corporativa e divulgação do gerenciamento de riscos corporativos estão positiva e significativamente relacionadas com o desempenho financeiro das empresas. Estes achados revelam uma lacuna de pesquisa, principalmente na literatura nacional, e instigam a realização de novas pesquisas com caráter diagnóstico e relacional.

Diante desse contexto, o presente estudo busca responder a seguinte questão de pesquisa: Qual a associação da evidenciação da gestão de riscos com governança corporativa e desempenho de empresas brasileiras com American Depositary Receipt (ADRs)? Assim, o objetivo do estudo é analisar a associação da evidenciação do gerenciamento de riscos com governança corporativa e desempenho de empresas brasileiras com ADRs.

Apesar da incipiente literatura identificada sobre evidenciação de gerenciamento de riscos, esta pesquisa é subsidiada por estudos que fizeram alguma incursão na temática. O estudo de Zonatto e Beuren (2010) fornece categorias de riscos para determinar o índice da evidenciação da gestão de riscos nos Relatórios da Administração das empresas. Dessa forma, ressalvada a distinta técnica estatística aplicada neste estudo, pode-se corroborar os seus achados. Okafor e Ibadin (2011) analisaram a relação entre as variáveis divulgação do gerenciamento de riscos, governança corporativa e desempenho corporativo em empresas nigerianas, cujos resultados podem reforçar os achados associativos do presente estudo e apontar novas lacunas de pesquisa.

Em relação à pesquisa de Elshandidy e Neri (2015), inova-se com a mensuração do nível de evidenciação dos riscos, uma vez que estes autores fizeram uso somente de um escore ao investigar empresas italianas. Aqui faz-se uma abordagem diferenciada sobre a evidenciação da gestão de riscos, baseada exclusivamente na divulgação agregada da gestão de riscos, visto que pesquisas internacionais têm destacado que a evidenciação da gestão de riscos tem sido incorporada no processo decisório (Campbell et al., 2014; Elshandidy \& Neri, 2015). Além disto, Jorgensen e Kirschenheiter (2003) advertem que, se uma empresa optar por não divulgar informações de risco, ela terá um prêmio de risco mais elevado do que as que fornecem estas informações, e este prêmio provavelmente será maior na presença de divulgação obrigatória de riscos do que a divulgação voluntária dos mesmos.

Estudos como o de Wang e Hussainey (2013) e Elshandidy e Neri (2015) apresentaram abordagens para a evidenciação do discurso de prospecção e evidenciação da gestão de riscos, respectivamente. Estes autores apresentaram que a evidenciação, tanto do discurso de prospecção da organização como da gestão de riscos, está intimamente relacionada com a governança corporativa e o desempenho. Desse modo, com parcimônia, argumenta-se que este estudo é contemporâneo e inovador ao tangenciar estas abordagens no campo nacional, até então investigado em empresas de outros países. Analisar as empresas com ADRs também pode contribuir para o campo de pes- 
quisa, uma vez que contrastam quanto ao seu nível de internacionalização.

A contribuição deste estudo para a prática gerencial está no uso da evidenciação como um meio de comunicação com o mercado. A evidenciação do gerenciamento de riscos pode subsidiar a tomada de decisão de investidores. Sob a ótica da governança corporativa, a evidenciação dos riscos organizacionais é tratada como um limitador da transferência de recursos pelos controladores, tornando-os mais evidentes aos minoritários. No que tange ao desempenho, especula-se que maior desempenho empresarial impulsiona a divulgação sobre o gerenciamento de riscos, uma vez que a performance da empresa no mercado de capitais é fruto da confiança e da sua saudabilidade econômico-financeira, portanto, está imbricada no relacionamento com os financiadores da atividade operacional que apostam nos riscos assumidos pelos gestores, visto o nível de transparência estabelecido nesta relação.

\section{FUNDAMENTO}

\subsection{Evidenciação da Gestão de Riscos}

A gestão de riscos deixou de ser uma preocupação exclusiva das organizações financeiras e passou a ser também de interesse das empresas não financeiras, em especial aquelas líderes dos seus segmentos de atuação (Saito \& Schiozer, 2007). O interesse dessas empresas tornou-se mais proeminente quando foram noticiados escândalos de grandes corporações norte-americanas e de outras nações, os quais ameaçaram a continuidade dos negócios, chamando a atenção de investidores, credores, gestores, governos e da academia.

Outro fato que contribuiu para a expansão da temática da gestão de riscos para organizações não financeiras é o entendimento de que a tesouraria destas grandes corporações atua como uma instituição financeira. De acordo com Hoji (2001), é uma das partes pertencentes ao organograma da empresa no qual os recursos financeiros transitam. O autor aponta ainda que a finalidade básica da tesouraria é assegurar os recursos e instrumentos financeiros necessários à manutenção e viabilidade dos negócios da empresa.

Duarte Jr (2001), Farrell (2004), Guimarães et al. (2009), Arena et al. (2010) e Zonatto e Beuren (2010) analisaram a gestão de riscos e suas interfaces corporativas. Farrell (2004), por exemplo, recomenda o uso de estruturação de controles de riscos a partir da adoção de metodologias aceitáveis e conhecidas mundialmente, como a do Committee of Sponsoring Organizations of the Treadway Commission, COSO 1 (Internal Control Framework) ou COSO 2 (Enterprise Risk Management). No entanto, não há consenso na literatura quanto aos riscos corporativos que as empresas estão expostas. As menções mais recorrentes apontam para: riscos de crédito (Altman, Caouette \& Narayanan, 2000), riscos legais, riscos operacionais (Duarte Jr, 2001), riscos de mercado (Duarte Jr, 2001; Marshall, 2002; COSO, 2004), risco estratégico (COSO, 2004), risco de liquidez (COSO, 2004) e risco de imagem (COSO, 2004). Nesse sentido, a evidenciação da gestão de riscos é uma oportunidade para a empresa apresentar ao mercado os riscos a que está exposta e seu posicionamento quanto a tais riscos.

Diante desse cenário, as empresas são guiadas por objetivos no que tange à prática da evidenciação da gestão de riscos. Uma forma de observar esse incentivo é recorrer à taxonomia para pesquisas sobre evidenciação na área contábil proposta por Verrecchia (2001), cujo quadro conceitual expõe que a prática da evidenciação é conduzida por três olhares: (i) pesquisa sobre divulgação baseada na associação; (ii) pesquisa sobre divulgação baseada no julgamento; e (iii) pesquisa sobre divulgação baseada na eficiência.

A primeira abordagem versa sobre o efeito das divulgações exógenas de alterações cumulativas ou ininterruptas do indivíduo, principalmente pelo comportamento dos preços de ativos. Alencar (2007, p. 17) aduz que é "uma versão estilizada da relação entre disclosure e mudança de preço na qual esta é explicada apenas pela informação advinda diretamente do disclosure e em um 
mundo onde não ocorrem transações".

A segunda abordagem examina como os gestores e ou as empresas descrevem a sua relação com a divulgação de informações por eles já conhecidas, ou seja, como ocorre a divulgação de informações que os mesmos detêm. Alencar (2007, p. 17) expõe que "a discricionariedade do disclosure estuda o problema de seleção adversa inerente ao mercado de capitais que leva o vendedor (da ação) a abrir todas as informações".

A última abordagem elencada por Verrecchia (2001) discute mecanismos de divulgação, quais configurações de divulgações são preferidas na ausência de conhecimento prévio sobre a informação. Alencar (2007) aduz que neste escopo se detém com mais detalhes a ligação entre disclosure e eficiência, abordando a questão do componente de assimetria informacional no custo de capital próprio.

Portanto, relacionando a configuração desta pesquisa com a estrutura proposta por Verrecchia (2001), os seus resultados podem ser visualizados a partir da segunda taxonomia, pois considera a evidenciação do gerenciamento de riscos como um processo endógeno, e a associação desta com a governança corporativa e do desempenho empresarial, que podem ser entendidos como enforcements que conduzem à configuração da divulgação das empresas.

\subsection{Governança Corporativa}

A governança corporativa, de acordo com o Instituto Brasileiro de Governança Corporativa (IBGC, 2009), é um sistema pelo qual as sociedades são dirigidas e controladas. A estrutura de governança corporativa especifica a distribuição de direitos e responsabilidades entre os diferentes participantes da empresa - diretoria, gerentes, acionistas e outras partes interessadas - e explicita as regras e procedimentos para a tomada de decisões corporativas. Segundo a Organização para a Cooperação e Desenvolvimento Econômico (OCDE, 1999), ao fazer isto fornece a estrutura pela qual os objetivos da empresa são definidos e os meios para alcançar estes objetivos e acompanhar o desempenho.

Depreende-se que a governança corporativa é um mecanismo de gestão que visa mitigar a assimetria informacional existente entre proprietários e gestores. $\mathrm{O}$ conflito entre proprietários e gestores é abordado pela Teoria da Agência, que versa sobre o processo em que uma ou mais pessoas (principal) empregam outra pessoa (o agente) com o objetivo de executar uma tarefa ou serviço a seu favor. Esta relação envolve delegação hierárquica e autoridade de decisão para o agente. É nesse processo de delegação que o conflito pode ocorrer, o interesse pessoal frente à tomada de decisão, que na visão do principal é a mais adequada (Jensen \& Meckling, 1976).

A governança corporativa é percebida por Terra e Lima (2006) como um mecanismo utilizado pela empresa para diminuir a assimetria informacional entre agente e principal, atua no sentido de demonstrar aos acionistas que a empresa tem adotado medidas para que os recursos investidos por estes no financiamento da atividade operacional estão alinhados com os interesses tanto do agente como do principal no que tange à maximização da riqueza da empresa, tanto a curto como longo prazo. O desafio da governança corporativa é realizar o alinhamento dos interesses dos diversos atores envolvidos nas corporações, acionistas, gestores, empregados e credores (Terra \& Lima, 2006).

É neste contexto que a informação contábil mostra o seu valor (Terra \& Lima, 2006). Dependendo da forma como a mesma é socializada junto aos investidores, notam-se reações imediatas (Rizério, 2019). Pesquisas anteriores relacionaram diversas variáveis de governança corporativa com o disclosure, dentre elas pode-se citar a estrutura de propriedade (Eng \& Mak, 2003; Lanzana, 2004), a proporção de membros externos no conselho (Ho \& Wong, 2001; Eng \& Mak, 2003; Lanzana, 2004), a separação da figura do presidente da empresa e do presidente do conselho (Haniffa \& Cooke, 2002; Lanzana, 2004) e a existência de um comitê de auditoria (Ho \& Wong, 2001). 
No presente estudo, como mecanismos de governança corporativa consideraram-se duas variáveis: (i) direito de controle (percentual de ações ordinárias pertencentes ao acionista ou bloco controlador); e (ii) diferença entre direito de controle e direito sobre o fluxo de caixa do acionista controlador (diferença entre percentual de ações ordinárias pertencentes ao acionista controlador e percentual de ações totais pertencentes ao mesmo). A participação dos acionistas ordinários na governança corporativa é uma corrente da pesquisa que visa compreender a relação entre organização e sociedade (Silva, Santos \& Rodrigues, 2016).

\subsection{Desempenho Empresarial}

Para acompanhar o desempenho empresarial e dar suporte à tomada de decisões dos acionistas são usualmente consideradas informações de cunho contábil. Okafor e Ibadin (2011) utilizaram variáveis de desempenho, como Margem Bruta (GM), Retorno sobre o Patrimônio Líquido (ROE) e Retorno sobre o Ativo (ROA). Como variáveis de governança adotaram tamanho da empresa, composição do conselho, estrutura de propriedade, status dos diretores executivos e independência do comitê de auditoria, associando-os com divulgação da gestão de riscos. Os resultados mostraram que governança corporativa e divulgação da gestão de riscos estão positivamente relacionadas com GM e ROA.

O ROA mensura a relação entre lucro total e ativo. No entanto, Oyadomari (2008) alerta que o ROA não é tão perfeito, pois dependendo das políticas contábeis assumidas pela empresa no que tange ao reconhecimento de aplicações financeiras, estas podem estar sendo compensadas nas dívidas bancárias nos passivos, assim há forte recomendação, de quando do uso desta métrica, que seja considerado o ativo líquido. Dal Magro, Filipin e Fernandes (2015) consideraram como variável de desempenho a liquidez de mercado das empresas, calculando a média dos spreads relativos ao longo de um período de três meses.

A Margem Bruta (Gross Margin - GM) é um indicador da situação econômica da empresa, resulta da divisão do Lucro Bruto pelas Vendas Líquidas. Tal indicador apresenta quanto a empresa obtém de retorno sobre as vendas, após a dedução das despesas sobre vendas, como impostos sobre vendas, devoluções, abatimentos, custos sobre as mercadorias vendidas e serviços prestados (Matarazzo, 2010).

O Retorno sobre o Patrimônio Líquido (Return On Equity - ROE) representa uma avaliação das decisões tomadas pelos gestores quanto à gestão dos recursos próprios e de terceiros no período e verificar se o acionista foi beneficiado ou recompensado (Iudícibus, 1998). O autor aduz que uma das tarefas da administração é adotar ferramentas que possam garantir que os valores investidos pelos acionistas sejam direcionados para a construção de uma base sólida de fluxo de dividendos. A principal limitação deste indicador é quando a "empresa vem de sucessivos prejuízos, o que pode gerar elevadas taxas de retorno posteriores nos períodos em que o PL é sensivelmente reduzido; outra ressalva deve ser observada quando a empresa está em fase inicial do ciclo de vida" (Oyadomari, 2008, p. 21).

Assim, os atributos de desempenho empresarial que nortearam esta pesquisa são: (i) Margem Bruta (GM); e (ii) Retorno sobre o Patrimônio Líquido (ROE). Os atributos de desempenho empresarial selecionados versam sobre as competências operacional e financeira das empresas, incluindo a rentabilidade na ótica do acionista, embora possua limitações. De modo complementar, optou-se por utilizar o Ativo Total em sua forma logarítmica, como forma de mensurar o tamanho da empresa. A Teoria da Sinalização sugere que as empresas maiores têm mais seguidores e analistas, portanto, são mais capazes de distribuir informações firmes e em volume maior (Elshandidy \& Neri, 2015).

Neste sentido, utilizou-se a variável Ativo Total com a finalidade de reforçar os achados anteriores de pesquisas sobre evidenciação. Os achados podem corroborar os preceitos da Teoria da 
Sinalização. As relações encontradas a respeito da gestão de riscos e tamanho da empresa também podem ser incorporadas nas decisões dos gestores das empresas objeto de estudo. Assim, após a apresentação dos argumentos que reforçam a necessidade de desenvolvimento de pesquisas que relacionem o efeito da governança corporativa e do desempenho empresarial com a evidenciação da gestão de riscos, faz-se necessário detalhar a operacionalização da pesquisa.

\section{MÉTODO}

$\mathrm{Na}$ análise da associação da evidenciação do gerenciamento de riscos com governança corporativa e desempenho em empresas brasileiras com American Depositary Receipts (ADRs) diferentes procedimentos foram adotados. Quanto à evidenciação do gerenciamento de riscos analisou-se uma série histórica de Relatórios da Administração (RAs), do período de 2010 a 2014, das companhias abertas com ADRs. A análise de conteúdo foi pautada em categorias e subcategorias de análise de riscos. A composição do Índice de Evidenciação do Gerenciamento de Riscos (IEGR) deu-se a partir de uma escala dicotômica e uso da entropia informacional para identificar o conteúdo informacional. No que concerne às variáveis de governança corporativa e desempenho das empresas fez-se a coleta na base de dados da Economática ${ }^{\circledR}$ e em relatórios financeiros divulgados pelas empresas. De posse destes dados foi possível determinar o IEGR e as variáveis governança corporativa e desempenho das empresas. Na sequência analisou-se a associação entre as variáveis por meio da aplicação da técnica de correlação.

\subsection{População e Amostra}

A população da pesquisa compõe-se das 43 empresas com ADRs, sendo que quatro empresas do setor financeiro foram excluídas e duas empresas que não apresentaram os dados completos no período analisado. Assim, a amostra compreendeu 37 empresas. Inicialmente coletaram-se os RAs das empresas, do período de 2010 a 2014, no site da Brasil Bolsa Balcão [B] ${ }^{3}$. Este período compreendeu um processo de recuperação econômica vivenciado pelo país após a crise financeira global de 2008. Tal período foi caracterizado por três políticas econômicas principais (Bastos, 2017): queda da taxa de juros, com o objetivo de reduzir a atratividade de aplicações em títulos da dívida pública pelas empresas não financeiras e reduzir o custo para capital de giro e financiamento do investimento produtivo; aumento da taxa de câmbio, com o objetivo de aumentar a competitividade internacional da produção local no mercado interno e nas exportações, induzindo investimentos produtivos, sobretudo na indústria de transformação; e aumento da taxa de lucro, que objetivava a redução do custo financeiro da dívida pública para reduzir custos do investimento privado com oferta pública de subsídios creditícios, fiscais e trabalhistas.

\subsection{Categorias de análise}

No Quadro 1 apresentam-se as categorias de análise e as respectivas subcategorias de risco consideradas neste estudo.

Quadro 1 - Categorias e subcategorias de análise de riscos

\begin{tabular}{|c|c|c|}
\hline Categorias & Subcategorias & Descrição \\
\hline \multirow{3}{*}{$\begin{array}{l}\text { Riscos Ope- } \\
\text { racionais }\end{array}$} & $\begin{array}{l}\text { Risco organizacional } \\
\text { (RO_RO) }\end{array}$ & $\begin{array}{l}\text { Relativo à uma organização ineficiente, administração inconsistente e sem } \\
\text { objetivos de longo prazo definidos, fluxo de informações internos e exter- } \\
\text { nos deficientes, responsabilidades mal definidas, fraudes, acesso a informa- } \\
\text { ções internas por parte de concorrentes, etc. (Duarte Jr., 2001). }\end{array}$ \\
\hline & $\begin{array}{l}\text { Risco de operações } \\
\qquad\left(\mathrm{RO} \_ \text {ROP }\right)\end{array}$ & $\begin{array}{l}\text { Decorrente da falta de consistência e adequação dos sistemas de controle } \\
\text { interno, sistemas de processamento e informações, o que pode ocasionar } \\
\text { perdas inesperadas para a organização }\end{array}$ \\
\hline & Risco de pessoal (RO_RP) & $\begin{array}{l}\text { Problemas com empregados não qualificados e/ou pouco motivados, per- } \\
\text { sonalidade fraca, falsa ambição, carreiristas, etc (Duarte Jr., 2001). }\end{array}$ \\
\hline Riscos de & Risco do mercado acioná- & Provocado por fatores de mercado, como o IBOVESPA (Duarte Jr., 2001). \\
\hline
\end{tabular}




\begin{tabular}{|c|c|c|}
\hline \multirow[t]{4}{*}{ Mercado } & rio (RM_RMA) & \\
\hline & $\begin{array}{l}\text { Risco do mercado de câm- } \\
\text { bio (RM_RMC) }\end{array}$ & $\begin{array}{l}\text { Decorrente de assumir posições em moeda diferente da moeda local (Duar- } \\
\text { te Jr., 2001). }\end{array}$ \\
\hline & $\begin{array}{l}\text { Risco do mercado de juros } \\
\qquad \text { (RM_RMJ) }\end{array}$ & $\begin{array}{l}\text { Exposição à condição financeira de um banco, como movimentos adversos } \\
\text { nas taxas de juros, que afeta os ganhos da empresa e/ou valor econômico } \\
\text { dos ativos (Bank for International Settlements - BIS, 1997). }\end{array}$ \\
\hline & $\begin{array}{l}\text { Risco do mercado de com- } \\
\text { modities (RM_RMC) }\end{array}$ & $\begin{array}{l}\text { Exposição do contrato estabelecido a fatores externos, como climáticos, } \\
\text { tecnológicos, mão de obra, entre outros (Duarte Jr., 2001). }\end{array}$ \\
\hline \multirow{3}{*}{$\begin{array}{l}\text { Riscos de } \\
\text { Crédito }\end{array}$} & Risco do país (RC_RP) & $\begin{array}{l}\text { Associado ao ambiente econômico, social e político do país de origem do } \\
\text { tomador de crédito (Bank for International Settlements - BIS, 1997). }\end{array}$ \\
\hline & Risco político (RC_RPOL) & $\begin{array}{l}\text { Contingencial entre o fornecedor e o cliente (Caouette, Altman \& Naraya- } \\
\text { nan, 1999) }\end{array}$ \\
\hline & $\begin{array}{l}\text { Risco da falta de pagamen- } \\
\text { to (RC_RFP) }\end{array}$ & $\begin{array}{l}\text { Decorrente da inadimplência da contraparte de acordo com uma disposição } \\
\text { contratual (Bank for International Settlements - BIS, 1997). }\end{array}$ \\
\hline \multirow[b]{2}{*}{ Riscos Legais } & Risco insolvência (RL_RI) & $\begin{array}{l}\text { Possibilidade de perda na organização por insolvência de dispositivos le- } \\
\text { gais ou regulamentares (Zonatto \& Beuren, 2010). }\end{array}$ \\
\hline & Risco ilegalidade (RL_RIL) & $\begin{array}{l}\text { Questionamentos jurídicos ou fiscais, que podem ocasionar na organização } \\
\text { o desembolso de recursos a tais órgãos ou a terceiros (Zonatto \& Beuren, } \\
\text { 2010). }\end{array}$ \\
\hline \multirow{5}{*}{$\begin{array}{l}\text { Risco Estra- } \\
\text { tégico }\end{array}$} & Risco de estrutura (RE_RE) & $\begin{array}{l}\text { Relacionado à adoção de uma postura mecanicista ou orgânica quanto a } \\
\text { interface do fluxo de informação (Duarte Jr., 2001). }\end{array}$ \\
\hline & Risco de processo (RE_RP) & $\begin{array}{l}\text { Risco de praticar novas ideias, para atingir resultados desejados, por pesso- } \\
\text { as que mantém relações com outros e que provocam mudanças nos contex- } \\
\text { tos institucionais e organizacionais (Duarte Jr., 2001). }\end{array}$ \\
\hline & $\begin{array}{l}\text { Risco de pesquisa e desen- } \\
\text { volvimento (RE_RP\&D) }\end{array}$ & $\begin{array}{l}\text { Risco de implementar uma estratégia malsucedida ou ineficaz que fracasse } \\
\text { em alcançar os retornos pretendidos (Marshall, 2002) }\end{array}$ \\
\hline & $\begin{array}{l}\text { Risco de formação de cus- } \\
\text { tos (RE_RFC) }\end{array}$ & $\begin{array}{l}\text { Relacionado à adoção de um método de composição de custos na busca de } \\
\text { vantagem competitiva (Zonatto \& Beuren, 2010). }\end{array}$ \\
\hline & $\begin{array}{l}\text { Risco de formação do pre- } \\
\text { ço de venda (RE_RFV) }\end{array}$ & $\begin{array}{l}\text { Relacionado à obtenção de vantagem competitiva por meio do preço de } \\
\text { venda (Zonatto \& Beuren, 2010). }\end{array}$ \\
\hline \multirow{3}{*}{$\begin{array}{l}\text { Risco de } \\
\text { Liquidez }\end{array}$} & $\begin{array}{l}\text { Risco de investimentos } \\
\text { (RL_RI) }\end{array}$ & $\begin{array}{l}\text { Risco de alguma incerteza ou variável imprevista impactar nas operações } \\
\text { de mercado (COSO, 2004; Zonatto \& Beuren, 2010). }\end{array}$ \\
\hline & $\begin{array}{l}\text { Risco de impossibilidade } \\
\text { de obter financiamentos } \\
\text { (RL_RIOF) }\end{array}$ & $\begin{array}{l}\text { Decorrente da impossibilidade de obtenção de financiamentos (COSO, 2004; } \\
\text { Zonatto \& Beuren, 2010). }\end{array}$ \\
\hline & $\begin{array}{l}\text { Risco de insuficiência de } \\
\text { recursos (RL_RIR) }\end{array}$ & $\begin{array}{l}\text { Caracterizado pela insuficiência de recursos disponíveis para o cumprimen- } \\
\text { to de obrigações (COSO, 2004; Zonatto \& Beuren, 2010). }\end{array}$ \\
\hline \multirow{2}{*}{$\begin{array}{l}\text { Risco de } \\
\text { Imagem }\end{array}$} & $\begin{array}{c}\text { Risco da veiculação da } \\
\text { informação (RI_RVI) }\end{array}$ & $\begin{array}{l}\text { Decorrente da veiculação da informação que pode afetar a imagem da or- } \\
\text { ganização (COSO, 2004; Zonatto \& Beuren, 2010). }\end{array}$ \\
\hline & $\begin{array}{l}\text { Risco da postura ou má } \\
\text { conduta dos empregados } \\
\text { (RI_RVI) }\end{array}$ & $\begin{array}{l}\text { Decorrente da postura ou má conduta dos empregados. Um empregado } \\
\text { que rouba ou trapaceia pode transmitir a imagem de que isto é uma prática } \\
\text { da empresa (COSO, 2004; Zonatto \& Beuren, 2010) }\end{array}$ \\
\hline
\end{tabular}
Fonte: Elaboração própria.

Com as informações apresentadas no Quadro 1, foi realizada a análise categórica nos RAs para identificar os grupos de riscos reportados pelas empresas. A análise categórica deu-se de forma dicotômica, sendo 1 quando se identificava a presença da subcategoria de análise nos RAs e 0 caso contrário. Esse tipo de categorização é comum em pesquisas na área contábil (Wang \& Hussainey, 2013; Elshandidy \& Neri, 2015; Suzart, 2016). A partir do levantamento foi organizado por meio do software Microsoft Office Excel ${ }^{\circledR}$ um banco de dados com as empresas da amostra e as categorias e subcategorias da evidenciação da gestão de riscos identificadas no RAs de cada ano. A determinação do Índice de Evidenciação da Gestão de Riscos (IEGR) consistiu de três etapas: (i) soma dos valores obtidos em cada um dos itens das categorias de análise; (ii) contagem do número de itens considerados aplicáveis para cada empresa e; (iii) divisão da soma dos itens pelo número de itens aplicáveis. O escore foi obtido em termos percentuais em consonância com Suzart (2016). 
Além da composição do IEGR, aplicou-se nos itens das categorias de análise a técnica de entropia da informação. A entropia é uma medida da quantidade de informação, aplicável para avaliar um escopo onde há diferentes constatações (Zeleny, 1982). Segundo o autor, as respostas são mensuradas com base em um ponto de referência, dessa forma, cada constatação terá uma probabilidade dentro de um conjunto, de modo que constatações idênticas as esperadas não representem surpresas. As que surpreendem, por estarem distantes do que era esperado, avaliam a quantidade de informação contida em uma resposta ou em um conjunto de categorias (Santos, Dorow \& Beuren, 2016). O cálculo da entropia da informação para as subcategorias de gestão de riscos evidenciadas seguiu os procedimentos propostos por Zeleny (1982).

Sejam $d_{i}=\left(d_{i}^{1}, d_{i}^{2}, \ldots, d_{i}^{m}\right)$ os valores normalizados, em que $d_{i}^{k}=\frac{x_{i}^{k}}{x_{i}^{*}}$ caracteriza o conjunto $\mathrm{D}$, em termos do i-ésimo atributo.

Define-se $\mathrm{D}_{\mathrm{i}}=\sum_{\mathrm{k}=1}^{\mathrm{m}} \mathrm{d}_{\mathrm{i}}^{\mathrm{k}} ; \mathrm{i}=1,2, \ldots, \mathrm{n}$. A medida de entropia do contraste de intensidade para $\mathrm{o}$ i-ésimo atributo é calculado por $\mathrm{e}\left(\mathrm{d}_{\mathrm{i}}\right)=-\alpha \sum_{\mathrm{k}=1}^{\mathrm{m}} \frac{\mathrm{d}_{\mathrm{i}}^{\mathrm{k}}}{\mathrm{D}_{\mathrm{i}}} \operatorname{Ln}\left(\frac{\mathrm{d}_{\mathrm{i}}^{\mathrm{k}}}{\mathrm{D}_{\mathrm{i}}}\right)$, em que $\alpha=\frac{1}{\mathrm{e}_{\max }}>0$ e emax $=\operatorname{Ln}(\mathrm{m})$. Lembrando ainda que $0 \leq \mathrm{d}_{\mathrm{i}}^{\mathrm{k}} \leq 1 \mathrm{e}^{\mathrm{d}_{\mathrm{i}}^{\mathrm{k}} \geq 0}$.

Caso todos os $d_{i}^{k}$ forem iguais para um dado i, então $\frac{d_{i}^{k}}{D_{i}}=\frac{1}{n}$ e e(di) assume o valor máximo, isto é, emax $=\operatorname{Ln}(\mathrm{m})$. Ao se fixar $\alpha=\frac{1}{\mathrm{e}_{\max }}$, determina-se $0 \leq \mathrm{e}\left(\mathrm{d}_{\mathrm{i}}\right) \leq 1$ para todos os di's. Essa normalização é necessária para fins comparativos.

A entropia total de D é definida por: $E=\sum_{i=1}^{n} e\left(d_{i}\right)$. Quanto maior for e(di), menor é a informação transmitida pelo i-ésimo atributo, menos dispersa se encontra a informação. Caso e(di) $=$ emax $=\operatorname{Ln}(m)$, então o i-ésimo atributo não transmite informação e essa pode ser removida da análise. Devido ao peso $\tilde{\lambda}_{i}$ ser inversamente relacionado a e(di), usa-se 1-e(di) ao invés de e(di) e normaliza-se para assegurar que $0 \leq \tilde{\lambda}_{\mathrm{i}} \leq 1 \mathrm{e} \mathrm{e}_{\mathrm{i}=1}^{\sum^{\mathrm{n}} \tilde{\lambda}_{\mathrm{i}}=1}$. Assim, a entropia da informação pode ser representada por: $\tilde{\lambda}_{\mathrm{i}}=\frac{1}{\mathrm{n}-\mathrm{E}}\left[1-\mathrm{e}\left(\mathrm{d}_{\mathrm{i}}\right)\right]=\frac{\left[1-\mathrm{e}\left(\mathrm{d}_{\mathrm{i}}\right)\right]}{\mathrm{n}-\mathrm{E}}$.

\subsection{Variáveis de governança corporativa e desempenho}

No Quadro 2 são apresentadas as variáveis de governança corporativa e de desempenho empresarial, com as respectivas operacionalizações. 
Quadro 2 - Variáveis de governança corporativa e de desempenho empresarial

\begin{tabular}{|c|c|c|c|}
\hline Construtos & Variáveis & Operacionalização & Referências \\
\hline \multirow[b]{2}{*}{$\begin{array}{l}\text { Governança } \\
\text { Corporativa }\end{array}$} & $\begin{array}{c}\text { Direito de controle } \\
(\mathrm{BLOC}) \\
\end{array}$ & $\begin{array}{l}\text { Percentual de ações ordinárias pertencentes ao acionista ou } \\
\text { bloco controlador. }\end{array}$ & \multirow[b]{2}{*}{ Lanzana (2004) } \\
\hline & $\begin{array}{l}\text { Diferença entre direi- } \\
\text { to de controle e direi- } \\
\text { to sobre o fluxo de } \\
\text { caixa do acionista } \\
\text { controlador (DIF) }\end{array}$ & $\begin{array}{l}\text { Diferença entre o percentual de ações ordinárias perten- } \\
\text { centes ao acionista controlador e o percentual de ações } \\
\text { totais pertencentes ao mesmo. }\end{array}$ & \\
\hline \multirow{3}{*}{$\begin{array}{l}\text { Desempenho } \\
\text { Empresarial }\end{array}$} & Margem Bruta (GM) & $\mathrm{GM}=\frac{\text { Lucro Bruto }}{\text { Receita Operacional Líquida }}$ & \multirow[b]{2}{*}{$\begin{array}{l}\text { Okafor e Ibadin } \\
\qquad(2011)\end{array}$} \\
\hline & $\begin{array}{l}\text { Retorno sobre o Pa- } \\
\text { trimônio Líquido } \\
\quad \text { (ROE) }\end{array}$ & $\mathrm{ROE}=\frac{\text { Lucro Líquido }}{\text { Patrimônio Líquido em } 31 \text { de dez. de X0 }}$ & \\
\hline & Ativo Total (AT) & $\mathrm{AT}=$ Logaritmo do Ativo Total & $\begin{array}{l}\text { Stickney e Weil } \\
\text { (2009) }\end{array}$ \\
\hline
\end{tabular}

Fonte: Elaboração própria.

A coleta de dados relativa ao desempenho empresarial e governança corporativa foi realizada na Economática ${ }^{\circledR}$ e em relatórios financeiros divulgados pelas empresas.

\subsection{Análise dos Dados}

Os dados foram submetidos a uma correlação. Para tal, aplicaram-se os testes de normalidade de Jarque-Bera e de Lilliefors, que revelaram que os dados não apresentam uma distribuição normal, as significâncias dos testes foram respectivamente p-valor 0,0038 e p-valor $\sim=0,03$. Dessa forma, foi aplicado o teste não-paramétrico coeficiente de correlação de postos de Spearman ou Correlação de Spearman. Ressalta-se que a aplicação da Correlação de Spearman deu-se a partir das médias sobre os indicadores e sobre os índices de evidenciação coletados. Esta ação é sustentada, visto que em separado e diante das médias os indicadores de significância foram os mesmos, revelando que tais indicadores não apresentaram volatilidade no período analisado e nem significância distinta.

\section{RESULTADOS}

\subsection{Evidenciação da Gestão de Riscos}

Inicia-se a descrição e análise de dados com a apresentação do Índice de Evidenciação da Gestão de Riscos por empresa e por período analisado, como disposto na Tabela 1.

Tabela 1 - Índice de Evidenciação da Gestão de Riscos por empresa e período

\begin{tabular}{l|c|c|c|c|c|c}
\hline \multicolumn{1}{c|}{ Empresas } & 2010 & 2011 & 2012 & 2013 & 2014 & Média \\
\hline AES Tietê & 0,182 & 0 & 0,136 & 0,136 & 0,136 & 0,118 \\
\hline Braskem & 0,136 & 0,136 & 0,318 & 0,136 & 0,136 & 0,173 \\
\hline BRF Foods & 0,136 & 0,182 & 0,273 & 0,227 & 0,364 & 0,236 \\
\hline Cemig & 0,227 & 0,318 & 0,091 & 0,136 & 0,273 & 0,209 \\
\hline CESP & 0,227 & 0,182 & 0,182 & 0,182 & 0,227 & 0,2 \\
\hline Cielo & 0 & 0,091 & 0,091 & 0,045 & 0,091 & 0,064 \\
\hline Copel & 0,182 & 0,273 & 0,227 & 0,5 & 0,5 & 0,336 \\
\hline Cosan & 0,227 & 0,091 & 0,136 & 0,136 & 0,045 & 0,127 \\
\hline CPFL & 0,136 & 0,091 & 0,091 & 0,182 & 0,091 & 0,118 \\
\hline CSN & 0,182 & 0,136 & 0,136 & 0,136 & 0,136 & 0,145 \\
\hline Cyrela Brasil & 0 & 0,136 & 0,091 & 0,045 & 0,045 & 0,064 \\
\hline Eletrobrás & 0,045 & 0 & 0 & 0 & 0 & 0,009 \\
\hline Embraer & 0,227 & 0,136 & 0,136 & 0,136 & 0,273 & 0,182 \\
\hline
\end{tabular}




\begin{tabular}{|c|c|c|c|c|c|c|}
\hline Fibria Celulose & 0,136 & 0,227 & 0,182 & 0,182 & 0,182 & 0,182 \\
\hline Gafisa & 0 & 0,045 & 0,091 & 0,091 & 0,182 & 0,082 \\
\hline Gerdau & 0,045 & 0,182 & 0,045 & 0,091 & 0 & 0,073 \\
\hline Gol & 0,182 & 0,091 & 0,091 & 0,091 & 0 & 0,091 \\
\hline Hypermarcas & 0,136 & 0,045 & 0 & 0 & 0 & 0,036 \\
\hline Iochpe Maxion & 0 & 0 & 0 & 0,045 & 0,091 & 0,027 \\
\hline JBS & 0,091 & 0 & 0 & 0,045 & 0,182 & 0,064 \\
\hline Klabin & 0,091 & 0 & 0 & 0 & 0,045 & 0,027 \\
\hline Média & 0,114 & 0,124 & 0,109 & 0,119 & 0,124 & 0,118 \\
\hline MMX & 0 & 0,045 & 0,045 & 0,136 & 0,091 & 0,064 \\
\hline Oi & 0 & 0,136 & 0,091 & 0,273 & 0,182 & 0,136 \\
\hline Pão de Açúcar & 0 & 0 & 0 & 0 & 0 & 0 \\
\hline PDG Realty & 0 & 0,182 & 0 & 0,182 & 0,091 & 0,091 \\
\hline Petrobras & 0,364 & 0,227 & 0,364 & 0,273 & 0,273 & 0,3 \\
\hline PRUMO Logística & 0 & 0 & 0 & 0 & 0 & 0 \\
\hline Rossi Residencial & 0,091 & 0,045 & 0 & 0,045 & 0 & 0,036 \\
\hline Sabesp & 0,409 & 0,227 & 0,318 & 0,364 & 0,227 & 0,309 \\
\hline Suzano Papel e Celulose & 0,045 & 0 & 0 & 0 & 0 & 0,009 \\
\hline Telefônica Brasil & 0,136 & 0,364 & 0,045 & 0 & 0,136 & 0,136 \\
\hline TIM Part. & 0 & 0 & 0,136 & 0,136 & 0,091 & 0,073 \\
\hline Tractebel Energia & 0,182 & 0,227 & 0,364 & 0,136 & 0,455 & 0,273 \\
\hline Transmissão Paulista & 0,045 & 0,091 & 0 & 0,045 & 0 & 0,036 \\
\hline Ultrapar Part. & 0,091 & 0,091 & 0,091 & 0 & 0 & 0,055 \\
\hline Usiminas & 0 & 0,182 & 0,182 & 0,182 & 0,045 & 0,118 \\
\hline Vale & 0,273 & 0,409 & 0,091 & 0,091 & 0 & 0,173 \\
\hline
\end{tabular}

Fonte: Dados da pesquisa.

Na Tabela 1, de modo geral não se observa um comportamento evolutivo dos índices nas empresas analisadas. Merecem destaque, no entanto, as empresas Copel, Sabesp, Petrobras, Tractebel Energia, BRF Foods, Cemig e CESP, pois apresentaram os maiores índices médios no período. Já as empresas com menores IEGR são: Transmissão Paulista, Iochpe Maxion, Klabin, Eletrobrás, Suzano Papel e Celulose, PRUMO Logística e Pão de Açúcar. A diversidade no nível de evidenciação pode sugerir que as empresas estão submetidas a contextos de riscos distintos.

Com a aplicação da entropia informacional foi operacionalizada a variável denominada neste estudo de Entropia da Informação da Gestão de Riscos (EIGR). Para os 22 eventos considerados, a amostra poderia atingir no máximo 22,000, isso se todos alcançassem o índice 1,000 de entropia da informação, portanto igual a escala de empresas com o grau máximo de evidenciação da gestão de riscos. Os resultados da entropia informacional, representada pela sigla e $\left(\mathrm{d}_{\mathrm{i}}\right)$, e o peso da questão estão demonstrados na Tabela 2.

Tabela 2 - Entropia informacional da evidenciação da gestão de riscos (EIGR)

\begin{tabular}{|c|c|c|c|c|c|c|c|c|c|c|c|}
\hline \multirow{2}{*}{\multicolumn{2}{|c|}{ EIGR }} & \multicolumn{2}{|c|}{2010} & \multicolumn{2}{|c|}{2011} & \multicolumn{2}{|c|}{2012} & \multicolumn{2}{|c|}{2013} & \multicolumn{2}{|c|}{2014} \\
\hline & & $e\left(d_{i}\right)$ & Peso & $e\left(d_{i}\right)$ & Peso & $e\left(d_{i}\right)$ & Peso & $e\left(d_{i}\right)$ & Peso & $\mathrm{e}\left(\mathrm{d}_{\mathrm{i}}\right)$ & Peso \\
\hline \multirow{4}{*}{$\mathrm{RO}$} & EIGR_RO_RO & 0,638 & 0,267 & 0,638 & 0,255 & 0,608 & 0,327 & 0,664 & 0,366 & 0,664 & 0,361 \\
\hline & EIGR_RO_ROP & 0,815 & 0,136 & 0,750 & 0,176 & 0,750 & 0,209 & 0,843 & 0,171 & 0,830 & 0,183 \\
\hline & EIGR_RO_RP & 0,192 & 0,596 & 0,192 & 0,569 & 0,446 & 0,464 & 0,576 & 0,463 & 0,576 & 0,456 \\
\hline & $\Sigma$ & 1,645 & 1,000 & 1,580 & 1,000 & 1,804 & 1,000 & 2,083 & 1,000 & 2,070 & 1,000 \\
\hline \multirow{5}{*}{$\mathrm{RM}$} & EIGR_RM_RMA & 0,192 & 0,287 & 0,192 & 0,282 & 0,000 & 0,329 & 0,192 & 0,282 & 0,384 & 0,225 \\
\hline & EIGR_RM_RMC & 0,496 & 0,179 & 0,496 & 0,176 & 0,576 & 0,140 & 0,496 & 0,176 & 0,496 & 0,184 \\
\hline & EIGR_RM_RMJ & 0,304 & 0,247 & 0,446 & 0,193 & 0,384 & 0,203 & 0,446 & 0,193 & 0,192 & 0,295 \\
\hline & EIGR_RM_RMC & 0,192 & 0,287 & 0,000 & 0,349 & 0,000 & 0,329 & 0,000 & 0,349 & 0,192 & 0,295 \\
\hline & $\Sigma$ & 1,184 & 1,000 & 1,134 & 1,000 & 0,960 & 1,000 & 1,134 & 1,000 & 1,264 & 1,000 \\
\hline $\mathrm{RC}$ & EIGR_RC_RP & 0,496 & 0,384 & 0,608 & 0,207 & 0,446 & 0,289 & 0,446 & 0,474 & 0,446 & 0,304 \\
\hline
\end{tabular}




\begin{tabular}{|c|c|c|c|c|c|c|c|c|c|c|c|}
\hline & $\begin{array}{l}\text { EIGR_RC_RPOL } \\
\text { EIGR_RC_RFP }\end{array}$ & 0,192 & $\begin{array}{r}0,616 \\
\text { N/A }\end{array}$ & $\begin{array}{l}0,496 \\
0,000\end{array}$ & $\begin{array}{l}0,266 \\
0,528\end{array}$ & $\begin{array}{l}0,446 \\
0,192\end{array}$ & $\begin{array}{l}0,289 \\
0,422\end{array}$ & 0,384 & $\begin{array}{r}0,526 \\
\text { N/A }\end{array}$ & $\begin{array}{l}0,539 \\
0,192\end{array}$ & $\begin{array}{l}0,253 \\
0,443\end{array}$ \\
\hline & $\Sigma$ & 0,688 & 1,000 & 1,105 & 1,000 & 1,083 & 1,000 & 0,830 & 1,000 & 1,177 & 1,000 \\
\hline \multirow{3}{*}{ RL } & EIGR_RL_RI & & N/A & 0,000 & 0,619 & 0,000 & 0,619 & & N/A & 0,000 & 0,684 \\
\hline & EIGR_RL_RIL & 0,384 & 1,000 & 0,384 & 0,381 & 0,384 & 0,381 & 0,384 & 1,000 & 0,539 & 0,316 \\
\hline & $\Sigma$ & 0,384 & 1,000 & 0,384 & 1,000 & 0,384 & 1,000 & 0,384 & 1,000 & 0,539 & 1,000 \\
\hline \multirow{6}{*}{ RE } & EIGR_RE_RE & 0,192 & 0,268 & 0,192 & 0,262 & 0,192 & 0,278 & 0,000 & 0,259 & 0,000 & 0,246 \\
\hline & EIGR_RE_RP & 0,608 & 0,130 & 0,731 & 0,087 & 0,710 & 0,100 & 0,638 & 0,094 & 0,638 & 0,089 \\
\hline & EIGR_RE_RP\&D & 0,496 & 0,167 & 0,304 & 0,226 & 0,000 & 0,344 & 0,304 & 0,180 & 0,000 & 0,246 \\
\hline & EIGR_RE_RFC & 0,304 & 0,231 & 0,304 & 0,226 & & N/A & 0,000 & 0,259 & 0,304 & 0,171 \\
\hline & EIGR_RE_RFV & 0,384 & 0,204 & 0,384 & 0,200 & 0,192 & 0,278 & 0,192 & 0,209 & 0,000 & 0,246 \\
\hline & $\Sigma$ & 1,985 & 1,000 & 1,915 & 1,000 & 1,094 & 1,000 & 1,134 & 1,000 & 0,942 & 1,000 \\
\hline \multirow{4}{*}{ RL } & EIGR_RL_RI & 0,000 & 0,448 & 0,192 & 0,372 & 0,304 & 0,500 & 0,192 & 0,372 & 0,000 & 0,356 \\
\hline & EIGR_RL_RIOF & 0,384 & 0,276 & 0,446 & 0,255 & 0,304 & 0,500 & 0,192 & 0,372 & 0,000 & 0,356 \\
\hline & EIGR_RL_RIR & 0,384 & 0,276 & 0,192 & 0,372 & & N/A & 0,446 & 0,255 & 0,192 & 0,288 \\
\hline & $\Sigma$ & 0,768 & 1,000 & 0,830 & 1,000 & 0,608 & 1,000 & 0,830 & 1,000 & 0,192 & 1,000 \\
\hline \multirow{3}{*}{ RI } & EIGR_RI_RVI & 0,384 & 1,000 & 0,384 & 1,000 & 0,446 & 1,000 & 0,384 & 1,000 & 0,384 & 0,433 \\
\hline & EIGR_RI_RVI & & N/A & & N/A & & N/A & & N/A & 0,192 & 0,567 \\
\hline & $\Sigma$ & 0,384 & 1,000 & 0,384 & 1,000 & 0,446 & 1,000 & 0,384 & 1,000 & 0,576 & 1,000 \\
\hline
\end{tabular}

Fonte: Dados da pesquisa.

Observa-se na Tabela 2 que os valores apresentam-se de forma distinta e distantes, indicando que há dispersão na amostra nas categorias analisadas. Nos anos considerados, o grupo mais representativo pela entropia encontrada nos anos de 2010 e 2011 foi o risco estratégico, que representa 1,985 e 1,915 dos riscos evidenciados, respectivamente. Já nos anos de 2012, 2013 e 2014 obteve maior representatividade a evidenciação do grupo riscos operacionais, com respectivamente os seguintes valores de entropia: 1,804, 2,083 e 2,070. Os grupos com maior entropia também apresentam menor dispersão de evidenciação do gerenciamento de riscos.

A evidenciação proveniente dos riscos operacionais, com a aplicação da técnica da entropia da informação, é a segunda dimensão com maior peso nos relatos. O grupo atingiu 1,645 e 1,580 nos anos de 2010 e 2011, respectivamente. Ainda na segunda dimensão, quanto ao peso nos relatos, nos anos de 2012 e 2013, merece destaque o grupo de riscos estratégicos, que na análise categórica pautada nas categorias de análise desta pesquisa, apresentou peso de entropia igual a 1,094 e 1,134, nesta ordem. Em 2013, o grupo de riscos de mercado apresentou peso de entropia 1,134, se equiparando ao peso dos riscos estratégicos.

Estes resultados mostram-se alinhados em parte com os de Zonatto e Beuren (2010), embora tenham aplicado um método estatístico diferente do que fora aplicado neste estudo. Os níveis de evidenciação do gerenciamento de riscos assemelham-se, uma vez que das 47,57\% categorias de riscos evidenciadas, ou seja, 82 observações identificadas nos RAs, 39\% dizem respeito aos riscos operacionais, legais e de mercado. Portanto, os riscos operacionais também se destacaram quanto ao seu nível de evidenciação. De certo modo, este cenário já era esperado. Os estudos comumente mostram que os gestores direcionam esforços para mitigar os riscos que podem prejudicar a operação da empresa.

Em geral, ao se analisar a entropia informacional e o peso da informação, observa-se que a evidenciação da gestão de riscos com menores dispersões está relacionada aos riscos estratégicos e operacionais. Por sua vez, as evidenciações com maiores dispersões estão relacionadas aos riscos legais e de imagem, ou seja, as empresas da amostra não evidenciam a prática do gerenciamento dos riscos relacionados a tais categorias. 


\subsection{Evidenciação da Gestão de Riscos, Governança Corporativa e Desempenho}

A correlação de Spearman foi aplicada considerando os dados das 37 empresas da amostra, computando a média dos indicadores elencados. Os resultados desta ação são apresentados na Tabela 3.

Tabela 3 - Correlação de Spearman entre as variáveis da pesquisa.

\begin{tabular}{|c|c|c|c|c|c|c|}
\hline Variáveis & IEGR & ROE & GM & BLOC & DIF & $\mathrm{AT}$ \\
\hline IEGR & 1,000 & & & & & \\
\hline $\mathrm{ROE}$ & 0,168 & 1,000 & & & & \\
\hline GM & $-0,090$ & 0,293 & 1,000 & & & \\
\hline BLOC & 0,070 & 0,038 & 0,238 & 1,000 & & \\
\hline DIF & $-0,037$ & $-0,119$ & 0,026 & $0,785^{* *}$ & 1,000 & \\
\hline $\mathrm{AT}$ & $0,379^{*}$ & $-0,086$ & $-0,187$ & 0,207 & 0,270 & 1,000 \\
\hline
\end{tabular}

Nota: Correlação significativa ao nível de ${ }^{*} 0,05 \mathrm{e}^{* *} 0,01$.

Fonte: Dados da pesquisa.

De modo geral, verifica-se na Tabela 3 que o índice de evidenciação do gerenciamento de riscos (IEGR) se correlacionou de forma positiva com o ativo total (AT) ao nível de significância de 0,05. Estes resultados convergem com o observado por Elshandidy e Neri (2015), de que empresas com maior porte ou maior liquidez tendem a ser mais transparentes em vista da sua visibilidade, acesso ao mercado de capitais e custos menores na geração de informações. As variáveis de governança corporativa, nesta pesquisa o Direito de Controle (BLOC) e a Diferença entre Direito de Controle e Direito sobre o Fluxo de Caixa do Acionista Controlador (DIF), estão correlacionadas ao nível de significância de 0,01 .

No que tange à associação do IEGR com as variáveis de governança corporativa, nota-se que não houve associação significante. Os resultados apresentados a esse respeito, portanto, não permitem inferir que quanto maior a concentração de ações com direito a voto nas mãos do acionista controlador (BLOC), menor é o nível de evidenciação do gerenciamento de riscos (IEGR). Isto em um cenário significante parece fazer sentido na medida em que estas empresas possuem menor incentivo para serem transparentes, já que seus acionistas controladores obtêm informações que não são públicas, diferente dos acionistas minoritários, que dependem dos relatórios publicados (Lanzana, 2004).

No que tange à diferença entre direito de controle e direito sobre o fluxo de caixa do acionista controlador (DIF) não se pode afirmar que quanto maior esta, maior o potencial de marginalização dos minoritários. Por vezes a governança corporativa pode inibir a divulgação de posturas e ações que buscam mitigar possíveis riscos, porém, a evidenciação do gerenciamento de riscos é também uma maneira de mostrar ao mercado que a empresa desenvolve mecanismos que mitigam possíveis influências negativas do ambiente e promovem a valorização da empresa no mercado em meio a estas contingências.

Diferente dos resultados desta pesquisa, Elshandidy e Neri (2015) identificaram efeito negativo da relação entre evidenciação da gestão de riscos e variáveis de governança corporativa. Esta divergência de cenários instiga o desenvolvimento de novas investigações com os gestores das empresas, pois tais constatações podem estar relacionadas ao comportamento e crenças dos mesmos sobre a evidenciação do gerenciamento de riscos. Tal fato merece especial atenção de pesquisadores no Brasil, visto que a diferença entre a posse de ações ordinárias (direito de controle) e a posse do total de ações (direito sobre o fluxo de caixa) por parte dos acionistas controladores pode apresentar implicações para o mercado de capitais. A estrutura de capital pode causar assimetria informacional frente à evidenciação do gerenciamento de riscos, principalmente em cenários de crises e adversidades. Tais relatórios são relevantes na tomada de decisões de investidores e viabilizam a valoração da empresa no mercado (Miihkinen, 2013). 
Quanto à associação do nível de evidenciação do gerenciamento de riscos com o desempenho das empresas analisadas, não é possível afirmar que existe associação significante para o período analisado. Assim, não se pode afirmar que quando o gestor tende a elevar suas metas de margem bruta (GM), o mesmo seja guiado por uma tendência de marginalizar os acionistas e/ou os usuários da informação contábil frente às decisões de evidenciação do gerenciamento de riscos. Isso indica que a evidenciação do gerenciamento de risco não é imbricada com a margem bruta da empresa, ou seja, as alterações no nível desta última não comprometem a percepção de credibilidade do usuário na empresa quanto à evidenciação dos riscos praticados.

Diferente do estudo de Elshandidy e Neri (2015), que observou a evidenciação do gerenciamento de riscos intimamente relacionada com a liquidez e rentabilidade das empresas, no presente estudo o Retorno sobre o Patrimônio Líquido (ROE) não se mostrou associado ao nível de evidenciação do gerenciamento de riscos. Uma relação positiva e significativa entre o nível de evidenciação do gerenciamento de riscos e o desempenho da empresa indicaria que quanto maior o desempenho da empresa, maior o nível de evidenciação. No entanto, os resultados desta pesquisa não convergem neste sentido.

As constatações deste estudo reforçam que há uma série de argumentos teórico-empíricos que sustentam existir benefícios no que tange à prática da evidenciação. Essa sustentação tem amparo em especial nas pesquisas de Zonatto e Beuren (2010), Okafor e Ibadin (2011), Miihkinen (2013) e Elshandidy e Neri (2015). No entanto, ao se analisar os resultados desta pesquisa parece haver resistência por parte das empresas em evidenciar as práticas de gerenciamento de riscos, mesmo obrigadas a adotar este mecanismo de acordo com a Lei Sarbanes-Oxley, as diretrizes do COSO (2004) e a Instrução CVM 480/2009.

Sob a ótica de Verrecchia (2001), não se pode afirmar que as empresas brasileiras com ADRs julgam divulgar informações sobre o gerenciamento de riscos a partir de elementos consubstanciados na governança corporativa e no desempenho empresarial. No entanto, reconhece-se que tal divulgação é um processo endógeno praticado pelas empresas analisadas, ao mesmo tempo em que representa a discricionariedade do gestor frente aos resultados de governança corporativa e ao desempenho da empresa.

Os resultados deste estudo revelam lacunas de pesquisa, uma vez que se cria um cenário que adiciona as variáveis de governança corporativa e desempenho empresarial como possíveis influenciadores do nível de evidenciação do gerenciamento de riscos. A configuração das narrativas das decisões sobre divulgação de gerenciamento de riscos nos relatórios da administração não se mostrou estar associada à estrutura de governança corporativa das empresas com ADRs e nem ao seu desempenho no período analisado.

\section{CONCLUSÃO}

Esta pesquisa revelou que a evidenciação do gerenciamento de riscos de empresas brasileiras com ADRs não está associada com a estrutura de governança corporativa e desempenho empresarial. A relação constatada do nível de evidenciação do gerenciamento de riscos com governança corporativa e desempenho empresarial coaduna com os achados na literatura (Elshandidy \& Neri, 2015), no que segue: (i) transparência - quanto maior o tamanho da empresa maior é o nível de evidenciação; e diverge dos insights quanto à (ii) governança corporativa - quanto maior a concentração de ações com direito a voto nas mãos do acionista controlador, menor é o nível de evidenciação do gerenciamento de riscos; e ao (iii) desempenho empresarial - quanto maior a margem bruta, menor é o nível de evidenciação do gerenciamento de riscos, e quanto maior o desempenho da empresa mensurado pelo ROE, maior é o nível de evidenciação do gerenciamento de riscos.

Os resultados contribuem para a discussão teórico-empírica sobre evidenciação do gerenciamento de riscos com governança corporativa e desempenho empresarial. Na perspectiva dos en- 
sinamentos de Verrecchia (2001), nota-se que a discricionariedade é exercida nas narrativas dos Relatórios da Administração e estas não recebem influência e julgamento da estrutura de governança corporativa e do desempenho empresarial. Pesquisas que busquem corroborar os resultados em estudos futuros podem aprimorar os achados desta investigação em empresas brasileiras com ADRs. A busca por uma estrutura ideal de evidenciação do gerenciamento de riscos, assim como a adequação das métricas de governança corporativa e do desempenho empresarial.

Os resultados deste estudo apresentam um cenário propício para o desenvolvimento de novas investigações. Neste sentido, recomenda-se a reaplicação deste estudo em outras empresas brasileiras, em especial todas as empresas listadas, grupos específicos como o IBRx50 ou IBRx100, excluindo também o setor financeiro, com a adição de métricas de desempenho distintas e que atendam propósitos organizacionais diferentes dos selecionados nesta pesquisa. Além disso, recomenda-se que futuros estudos analisem no escopo estatístico outras técnicas que possam estabelecer correlações não identificadas nesta pesquisa.

\section{REFERÊNCIAS}

Alencar, R. C. (2007). Nível de disclosure e custo de capital próprio no mercado brasileiro. 2007. Tese de Doutorado, Universidade de São Paulo, São Paulo, SP, Brasil.

Altman, E. I., Caouette, J. B., \& Narayanan, P. I. (2000) Gestão do risco de crédito: o próximo grande desafio financeiro. Rio de Janeiro: Qualitymark.

Arena, M., Arnaboldi, M., \& Azzone, G. (2010). The organizational dynamics of enterprise risk management. Accounting, Organizations and Society, 35(7), 659-675.

Bank for International Settlements (BIS). (1997). Core effective principles in banking supervision. Recuperado em agosto 22, 2015, de http://www.bis.org

Bastos, P. P. Z. (2017). Ascensão e crise do governo Dilma Rousseff e o golpe de 2016: poder estrutural, contradição e ideologia. Revista de Economia Contemporânea, elocation - e172129, núm. esp., 1-63.

Campbell, J. L., Chen, H., Dhaliwal, D. S., Lu, H. M., \& Steele, L. B. (2014). The information content of mandatory risk factor disclosures in corporate filings. Review of Accounting Studies, 19(1), 396-455.

Caouette, J. B., Altman, E. I. \& Narayanan, P. (1999) Gestão do risco de crédito: o próximo grande desafi$o$. Rio de Janeiro: Qualitymark.

Committee of Sponsoring Organizations of the Treadway Commission (COSO) (2004). Enterprise risk management: framework integrated. New York: ICPA.

Comissão de Valores Mobiliários. Instrução CVM 480, de 07 de dezembro de 2009. Dispõe sobre o registro de emissores de valores mobiliários admitidos à negociação em mercados regulamentados de valores mobiliários. Recuperado em 04 abr., 2016, de http://www.cvm.gov.br/legislacao/instrucoes/inst480.html

Dal Magro, C. B.; Filipin, R., \& Fernandes, F. C. (2015). Gestão de riscos: Análise da evidenciação de riscos nas concessionárias de rodovias listadas na Bovespa com base na metodologia COSO. ConTexto, 15(30), 57-75.

Duarte Jr, A. M. (2001). Riscos: definições, tipos, medição e recomendações para seu gerenciamento gestão de risco e derivativos. São Paulo: Atlas.

Elshandidy, T., \& Neri, L. (2015). Corporate governance, risk disclosure practices, and market li- 
quidity: Comparative evidence from the UK and Italy. Corporate Governance: An International Review, 23(4), 331-356.

Eng, L. L., \& Mak, Y. T. (2003). Corporate governance and voluntary disclosure. Journal of Accounting and Public Policy, 22(4), 325-345.

Farrell, J. (2004). Internal controls and managing enterprise-wide risks. The CPA Journal, 74(8), 1112.

Gordon, L. A., Loeb, M. P., \& Tseng, C. Y. (2009). Enterprise risk management and firm performance: A contingency perspective. Journal of Accounting and Public Policy, 28(4), 301-327.

Guimarães, I. C., Parisi, C., Pereira, A. C., \& Weffort, E. F. J. (2009). A importância da controladoria na gestão de riscos das empresas não-financeiras: Um estudo da percepção de gestores de risco e controllers. Revista Brasileira de Gestão de Negócios, 11(1), 260-275.

Haniffa, R., \& Cooke, T. (2002). Culture, corporate governance and disclosure in Malaysian corporations. Abacus, 38(3), 317-349.

Ho, S. S., \& Wong, K. S. (2001). A study of the relationship between corporate governance structures and the extent of voluntary disclosure. Journal of International Accounting, Auditing and Taxation, 10(2), 139-156.

Hoji, M. (2001). Práticas de tesouraria. São Paulo: Atlas.

Holmberg, P., \& Wolak, F. (2016). Electricity markets: Designing auctions where suppliers have uncertain costs. SSRN. Recuperado em maio 31, 2020, de https://papers.ssrn.com/sol3/papers.cfm?abstract_id=2705568

Instituto Brasileiro de Governança Corporativa (IBGC) (2007). Guia de orientação para o gerenciamento de riscos corporativos. São Paulo: IBGC.

Instituto Brasileiro de Governança Corporativa (IBGC) (2009). Código das Melhores Práticas de Governança Corporativa. São Paulo: IBGC.

Iudícibus, S. (1998). Análise de Balanços: análise da liquidez e do endividamento, análise do giro, rentabilidade e alavancagem financeira. São Paulo: Atlas.

Jensen, M. C., \& Meckling, W. H. (1976). Theory of the firm: Managerial behavior, agency costs and ownership structure. Journal of Financial Economics, 3(4), 305-360.

Jin, L., Chen, C., Wang, X., Yu, J., \& Long, H. (2020). Research on information disclosure strategies of electricity retailers under new electricity reform in China. Science of The Total Environment, 710(25), 1-7.

Jorgensen, B. N., \& Kirschenheiter, M. T. (2003). Discretionary risk disclosures. The Accounting Review, 78(2), 449-469.

Lanzana, A. P. (2004). Relação entre disclosure e governança corporativa das empresas brasileiras. Dissertação de Mestrado, Universidade de São Paulo, São Paulo, SP, Brasil.

Marshall, C. (2002). Medindo e gerenciando riscos operacionais em instituições financeiras. Rio de Janeiro: Qualitymark.

Martins, M. A. (2006). Avaliação de desempenho empresarial como ferramenta para agregar valor ao negócio. ConTexto, 6(10), 1-27.

Matarazzo, D. C. (2010) Análise financeira de balanços: abordagem gerencial. São Paulo: Atlas. 
Miihkinen, A. (2013). The usefulness of firm risk disclosures under different firm riskiness, investor-interest, and market conditions: New evidence from Finland. Advances in Accounting, 29(2), 312-331.

Okafor, C. A., \& Ibadin, P. O. (2011). Corporate governance and corporate performance in selected companies in Nigeria. Afro-Asian Journal of Finance and Accounting, 2(4), 333-348.

Organização para Cooperação e Desenvolvimento Econômico (OCDE) (1999). Principles of Corporate Governance. Recuperado em ago. 12, 2019, de https://www.oecdilibrary.org/docserver/9789264236882-

en.pdf?expires=1602710851\&id=id\&accname=guest\&checksum=2233B18A143B54126686B55A AD94478D

Oyadomari, J. C. T. (2008). Uso do sistema de controle gerencial e desempenho: um estudo em empresas brasileiras sob a ótica da VBR (Visão Baseada em Recursos). Tese de Doutorado, Universidade de São Paulo, São Paulo, SP, Brasil.

Raffournier, B. (1995). The determinants of voluntary financial disclosure by Swiss listed companies. European Accounting Review, 4(2), 261-280.

Rizério, L. (2019). Ação do Magazine Luiza (MGLU3) dispara 7\% após balanço indicar crescimento exponencial da empresa. Infomoney. Recuperado em maio 31, 2020, de https://www.infomoney.com.br/mercados/magazine-luiza-mglu3-surpreende-mais-uma-vezcom-crescimento-exponencial-e-acoes-saltam-7/

Saito, R., \& Schiozer, R. F. (2007). Uso de derivativos em empresas não-financeiras listadas em bolsa no Brasil. Revista de Administração, 42(1), 97-107.

Santos, J. G. C. D., \& Coelho, A. C. (2018). Value-relevance do disclosure: fatores e gestão de riscos em firmas brasileiras. Revista Contabilidade E Finanças, 29(78), 390-404.

Santos, V. D., Dorow, D. R., \& Beuren, I. M. (2016). Práticas gerenciais de micro e pequenas empresas. Revista Ambiente Contábil, 8(1), 153-186.

Silva, F. B., Santos, D. F. L., \& Rodrigues, S. V. (2016). Desempenho comparativo de empresas com governança corporativa entre os setores bancário e industrial. Revista Eletrônica do Mestrado em Administração da Universidade Potiguar -RAUnP, 8(2), 48-65.

Stickney, C., \& Weil, R. (2009). Contabilidade Financeira: introdução aos conceitos, métodos e usos. São Paulo: Cengage Learning.

Suzart, J. A. S. (2016). Índices de evidenciação: Quando um mais zero não é igual a um. Contabilometria, 3(1), 52-70.

Terra, P. R. S., \& Lima, J. B. N. (2006). Governança corporativa e a reação do mercado de capitais à divulgação das informações contábeis. Revista Contabilidade \& Finanças-USP, 17(42), 35-49.

Verrecchia, R. E. (2001). Essays on disclosure. Journal of Accounting and Economics, 32(1-3), 97-180.

Wang, M., \& Hussainey, K. (2013). Voluntary forward-looking statements driven by corporate governance and their value relevance. Journal of Accounting and Public Policy, 32(3), 26-49.

Zeleny, M. (1982). Multiple criteria decision-making. New York: McGraw-Hill.

Zonatto, V. C. S., \& Beuren, I. M. (2010). Categorias de riscos evidenciadas nos relatórios da administração de empresas brasileiras com ADRs. Revista Brasileira de Gestão de Negócios, 12(35), 141-155. 